\title{
High motility group box 1 (HMGB1) protein and its receptor for advanced glycation end products (RAGE) expression in chronic rhinosinusitis without nasal polyps
}

\author{
Karolina Dzaman $^{1 *}$, Mariola Zagor ${ }^{1 *}$, Marta Molinska-Glura² ${ }^{2}$ Antoni Krzeski ${ }^{1}$ \\ *Both authors contributed equally to this work \\ ${ }^{1}$ Department of Otolaryngology, Faculty of Medicine and Dentistry, Medical University \\ of Warsaw, Poland \\ ${ }^{2}$ Department of Computer Science and Statistics, University of Medical Sciences, Poznan, Poland
}

\begin{abstract}
Introduction. Chronic rhinosinusitis (CRS) affects $14 \%$ of the world population. The high motility group box 1 (HMGB1) protein triggers inflammation, cell proliferation and cell survival through its receptor for advanced glycation end products (RAGE) upon release from stressed or necrotic cells. The aim of the study was to analyze the expression and function of HMGB1 and RAGE in CRS, providing more information about HMGB1 signaling pathway in CRS, to determine its potential clinical significance.

Material and methods. Thirty-seven patients with CRS and 26 normal controls (NC) were enrolled in this study. Classification of disease severity using the SNOT-20 questionnaire, nasal endoscopy, CT scan, assessment of allergy status, microbiological and cytological analysis was performed in patients. Fresh sinus mucosa samples were obtained and analyzed by immunohistochemistry for HMGB1 and RAGE expression in epithelial cells. ELISA assay was performed to evaluate the concentration of HMGB1 in the patients' sera.

Results. No differences were found in HMGB1 immunoexpression between CRS patients and NC, however there was a highly significant difference in RAGE immunoexpression between both groups. There was a correlation between RAGE expression and number of tissue-infiltrating lymphocytes. Further, RAGE expression positively correlated with disease severity and a positive history for allergies.

Conclusions. Interaction of HMGB1 and RAGE might be relevant to CRS pathomechanisms leading to sinus mucosa hyperproliferation. CRS pathogenesis might be especially related to the RAGE overexpression correlated with disease severity and allergy. (Folia Histochemica et Cytobiologica 2015, Vol. 53, No. 1, 70-78)
\end{abstract}

Key words: rhinosinusitis; chronic inflammation; HMGB1; RAGE; IHC; serum

\section{Introduction}

The immune system has evolved to respond to exogenous components of microbes through pathogen-associated molecular patterns (PAMPs) detected by

\footnotetext{
Correspondence address: M. Zagor, M.D., Ph.D.

Department of Otolaryngology, Division of Dentistry

Medical University of Warsaw

Stepinska St. 19/25, Warsaw

tel.: +48 694012 224, fax: +48223186266

e-mail: popkom@interia.pl
}

pattern-recognition receptors (PRRs), and also to endogenous danger signals, or damage-associated molecular patterns (DAMPs). DAMPS are released by dying or necrotic cells and contribute to inflammation. High motility group box 1 protein (HMGB1) is a multifunctional nuclear protein and one of the well-known members of the DAMP family [1]. It is present within the nuclei of almost all eukaryotic cells and functions as a DNA chaperone that stabilizes nucleosome formation and promotes access to transcriptional factors that target specific genes. Upon its release from stressed or necrotic cells, HMGB1 acts as an ex- 
tracellular trigger of inflammation, cell proliferation, migration and survival, mainly through interactions with its receptor for advanced glycation end products (RAGE) [2]. RAGE ligands are either released during cellular stress (S100 proteins, HMGB1, nucleic acids), or generated during prolonged hyperglycemia and inflammation (AGE, amyloid) [3]. Due to an enhanced level of RAGE ligands in chronic disorders, this receptor is hypothesized to have a causative effect in a range of inflammatory diseases [3-5]. Though RAGE was first identified as the receptor for HMGB1, subsequently, toll-like receptors 2 (TLR2) and TLR4 have also been identified to be involved in HMGB1 signaling [6]. These interactions trigger activation of key signaling pathways involved in the regulation of innate and adaptive immunity [7], polarizing the immune response towards Th1 or Th2 and secretion of different cytokines. Chavakis et al. demonstrated that RAGE can directly regulate leukocyte recruitment by serving as a counter-receptor for $\beta 2$-integrin Mac-1 [8]. RAGE activation plays a role in various diseases, including sepsis, rheumatoid arthritis, diabetic nephropathy, atherosclerosis, neurological diseases and cancer [9-13]. RAGE receptor exists in the cells in two different forms: mRAGE (membrane bound, full-length molecule containing transmembrane and cytoplasmic domains) and sRAGE (soluble, shorter form). sRAGE derived via alternative mRNA splicing is known as esRAGE (endogenous secretory RAGE' or RAGE_v1), whereas SRAGE derived from the proteolytic cleavage of mRAGE is referred to as cleaved RAGE (cRAGE). The soluble sRAGE form is present in the in the extracellular space and can bind RAGE ligands in the circulation $[14,15]$.

Chronic rhinosinusitis (CRS) is one of the most common health concerns affecting $14 \%$ of the world's population. The prevalence of CRS has increased over the last decade and has become a serious health problem. CRS is associated with significant low quality of life index, comparable to levels noticed in patients with life-risk illnesses such as chronic obstructive pulmonary disease or congestive heart failure [16]. Although a number of contributing factors are recognized, the underlying cause and mechanism of this disease remain unknown, and definite curative treatment does not exist.

CRS comes in two different subtypes, namely CRS without polyps (CRSsNP) and CRS with polyps (CRSwNP). CRSwNP is diagnosed when nasal polyps are visible at an appropriate nasal endoscopic examination. Otherwise, the disease is classified to CRSsNP. In the European position paper on rhinosinusitis and nasal polyps (EPOS 2012) [17], the current pathogenesis of these two CRS subtypes has been discussed.
Current research focuses on epithelial/immune cell interactions, the biofilm hypothesis and the superantigen hypothesis. CRS may be associated with other diseases, especially allergies, asthma or aspirin-exacerbated respiratory disease (AERD). The standard diagnostic procedures include medical history, nasal endoscopy, CT-scans (computed tomography) of the paranasal sinus, and allergy testing for common inhalant allergens. The American Academy of Otolaryngology has recommended the Lund and Mackay system for CT staging of CRS [18]. The choice of CRS therapy depends upon symptom intensity and includes topical steroids, antibiotics and/or surgery [19, 20].

To the best of our knowledge, there is currently no data available on both HMGB1 and RAGE expression and function in CRS pathogenesis. Few authors assessed HMGB1 expression in CRS patients, however, RAGE expression in correlation with HMGB1 in tissue has yet not been studied [21-23]. Furthermore, in one paper HMGB1 expression was assessed mainly in the inflammatory infiltrates (or polyp tissue) [24] and not in epithelial cells. There is emerging interest in the role of molecules that bind HMGB1, including RAGE, in CRS. We assumed that HMGB1-RAGE signaling pathway may contribute to the CRS pathomechanisms. To test our hypothesis, we investigated expression of HMGB1 in the whole tissue including the epithelial cells and the stroma of sinonasal mucosa obtained from CRS patients vs. epithelial cells of nasal mucosa of normal controls. Given the fact that HMGB1-RAGE interactions are known to promote inflammation in various pathologies, we hypothesized that these interactions could also occur and play a critical role in CRS.

\section{Material and methods}

Patients and tissue collection. Fresh sinus mucosa samples were obtained during surgery of the osteomeatal complex in 37 individuals diagnosed with CRS without nasal polyps (CRS). Based on EPOS 2012 classification [17], the presence of clinical and radiological evidence of chronic rhinosinusitis and absence of polyps in nasal endoscopy examination was required for entry into this study. Table 1 summarizes characteristics of the subjects included in this study. The group of CRS patients included 19 males and 18 females patients (median age 42 years, range 15-71 years). The group of normal healthy controls (NC) comprised 18 men and 8 women (median age 40 years; range $16-69$ years). The control subjects had nasal structural deformities, without clinical and radiological evidence of chronic rhinosinusitis. Control sinus mucosa (C-SM) included non-inflammatory changed normal mucosa from osteomeatal complex region. The study was approved by the Local Ethics Committee, 
Table 1. Clinicopathological characteristics of chronic rhinosinusitis (CRS) patients and control group patients ${ }^{\mathrm{a}}$ included in this study

\begin{tabular}{|l|c|c|}
\hline Characteristics & $\begin{array}{c}\text { Controls } \\
\mathbf{n = 2 6}\end{array}$ & $\begin{array}{c}\text { CRS } \\
\mathbf{n = 3 7}\end{array}$ \\
\hline $\begin{array}{l}\text { Sex } \\
\text { Male } \\
\text { Female }\end{array}$ & $\begin{array}{c}18 \\
8\end{array}$ & 19 \\
\hline $\begin{array}{l}\text { Age } \\
\text { Range } \\
\text { Median }\end{array}$ & $16-69$ & $15-71$ \\
\hline Allergy & 40 & 42 \\
\hline Asthma & 2 & 10 \\
\hline NSAIDs ${ }^{b}$ allergy & 0 & 1 \\
\hline $\begin{array}{l}\text { Average CT Lund-Mackay } \\
\text { scores }\end{array}$ & 2 & 0 \\
\hline Average SNOT scores & $21.5 \pm 15.4$ & $23.6 \pm 19.5$ \\
\hline $\begin{array}{l}\text { Serum concentration } \\
\text { of IgE [IU/mL] }\end{array}$ & $60 \pm 8.8$ & $156 \pm 206.7$ \\
\hline Positive microbiology findings & $25 \%$ & $34 \%$ \\
\hline
\end{tabular}

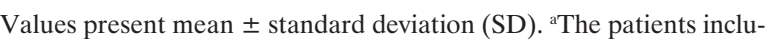
ded in the study received no oral steroid treatment for 2 months prior to surgery; ${ }^{b}$ NSAIDs — nonsteroidal anti-inflammatory drugs

Warsaw Medical University, Poland (KB 103/2012) and was compliant with the Helsinki Declaration. All participants signed an informed consent.

The classification of disease severity was performed using the SNOT-20 questionnaire (Sino-Nasal Outcome Test), nasal endoscopy and CT scan graded by Lund-Mackay scoring system $[18,25]$. We also recorded a number of sinus surgeries performed in CRS patients reflecting the recalcitrant sinus disease and intensity of CRS.

Allergy status. Allergy status was assessed based on the patient's history, a skin prick test and total $\operatorname{IgE}$ levels in patient's blood samples. NSAIDs allergy (nonsteroidal anti-inflammatory drugs allergy) and asthma were noted based on spirometry and medical anamnesis.

Microbiology. To determine the composition of sinonasal cultures, the middle meatus sample collection was performed in each patient under endoscopic control. Nasal swabbing from middle nasal meatus followed by agar culture identified the bacterial species present in the nasal cavity.

Cytology. To determine the population of inflammatory cells in the nasal cavity, cytology samples were taken under the middle turbinate. The cells were smeared on glass slides, fixed with alcohol, stained with hematoxylin and eosin
( $\mathrm{H} \& \mathrm{E})$ and evaluated under the light microscope. In the evaluation of the cytograms of each patient it was found that they could be classified to one of the six types of cytograms, previously described by Canakcioglu et al. [26].

Immunohistochemistry. Formalin-fixed and paraffin-embedded tissues sections were stained with $\mathrm{H} \& \mathrm{E}$ for light microscopy evaluation. The following primary antibodies were used for immunostaining of tissue sections: rabbit polyclonal anti-human HMGB1 diluted with Dako Antibody Diluent (1:1,000, Abcam, Cambridge, UK), rabbit polyclonal anti-human RAGE (1:100, LifeSpan Bioscience Inc., Seattle, WA, USA) and isotype control IgG (Dako, Gdynia, Poland). Paraffin sections of sinus mucosal tissue or normal mucosa were stained as previously described [27]. After standard deparaffinization, the EnVision + System (Dako) was used for staining according to the manufacturer's instructions. In short, after an overnight incubation with the primary antibodies (anti-human HMGB1, anti-human RAGE), sections were incubated with labeled polymer-horseradish peroxidase (HRP) anti-rabbit antibody and then with 3,3'-diaminobenzidine (Dako). To eliminate nonspecific binding sections were incubated with a serum-free protein blocker before adding the primary antibodies. Sections were counterstained with Meyer's hematoxylin and mounted in DPX (POCH, Gliwice, Poland). Slides were evaluated under the light microscope at total magnification $\times 400$ (Primo Star microscope, Carl Zeiss, Wetzlar, Germany). For digital image analysis, the software AnalySIS $\wedge \mathrm{B}$ (Camera Olypmus DP12, Melville, NY, USA) was used. All stained sections were analyzed and scored by two independent investigators (M.J.S. and K.D.) to avoid bias, and the two scores were averaged and recorded. The sections were scored according to the $\%$ of rhinosinusitis tissue staining ('positivity') $(<25 \%=0 ; 25-75 \%$ $=1$; and $>75 \%=2)$. The level of staining intensity was recorded as none $=0$, weak $=1$, moderate $=2$, or strong $=3$. As a positive control for HMGB1 and RAGE expression, sections of intestine and kidney were used, respectively.

Measurement of serum concentration of HMGB1. To evaluate the level of HMGB1 in the patients' sera, HMGB1 ELISA kit (U.S. Customers antibodies-online Inc, Atlanta, GA, USA) was used according to the manufacturers' instructions.

Statistical analysis. Data were summarized by descriptive statistics (Statistica 10.0, Statsoft, Cracow, Poland). Fisher's exact tests were used to determine if there was a difference in HMGB1 and RAGE expression among tissue types. Adjustments to $\mathrm{p}$-values were made using the Bonferroni step-down procedure. The ANOVA Kruskall-Wallis test was used to evaluate differences between groups. 


\section{Results}

\section{Cytology}

In 23 of the CRS patients a neutrophil cytogram was noticed, in 7 an injury cytograms, in 5 an eosinophil cytograms, in 1 a normal and in 1 a secretory cytograms. The control group showed normal nasal cytology in 14 cases, neutrophil and eosinophil cytograms in 4 cases and injure or secretory cytograms in 2 cases (Figure 1A2, 1A4).

\section{HMGB1 immunoexpression in tissues}

HMGB1 was detected in all tissues of normal controls and CRS patients. HMGB1 was localized in the nuclei and cytoplasm and in all cases staining intensity was evaluated as moderate or strong (Figure 1B1-4). We did not find statistically significant differences between NC vs. CRS in terms of HMGB1 immunoreactivity in the epithelial cells of sinonasal mucosa (Figure 1C; the epithelium lining marked with arrows and the stroma of sinus mucosa marked with stars).

No correlation was found between HMGB1 expression and disease severity evaluated based on SNOT, nasal endoscopy examination, CT scan and a number of surgery. Furthermore, we did not find any significant differences in the patients' cohort of HMGB1 expression and either the allergy or microbiological evaluation.

\section{RAGE expression in tissues}

RAGE was present in the cytoplasm of epithelial cells and inflammatory infiltrates. In CRS patients RAGE was detected in $94 \%$ of cases and its staining intensity ranged from weak to strong. RAGE was observed in $40 \%$ of NC, however, its expression was weak (Figure 1B5-8). Differences in RAGE expression between CRS and NC were highly significant $(\mathrm{p}<0.00005)$ (Figure 1D). In the CRS tissue, a moderate to large number of RAGE-immunopositive inflammatory cells were observed (data not shown).

\section{RAGE immunoexpression positively correlates with disease severity}

We observed significant differences in RAGE intensity and positivity depending on CT scan results (graded by the Lund-Mackay scoring system) in the CRS cohort (Figure 2). Staining intensity and positivity were stronger in patients with a higher $\mathrm{CT}$ score reflecting more extensive inflammatory changes in sinuses $(\mathrm{p}=0.0024$, $p=0.0163$, respectively). No significant differences in RAGE expression and severity of symptoms using the SNOT-20 questionnaire, or the number of previously performed surgeries in CRS patients or nasal endoscopy results were found.

\section{RAGE immunoexpression correlates with disease etiology}

We observed significantly stronger RAGE positivity in the sinus mucosa of patients with higher serum IgE values, who suffered from allergy, compared to patients with negative history for allergies (Figure $3 \mathrm{~A}, \mathrm{~B})$. We did not observe any correlation between RAGE expression, asthma or NSAIDs allergy. In this study, the microbiological content of the nasal cavity was associated with RAGE expression $(p=0.007)$ (Figure 3C) but there was no differences in the median value of RAGE expression and the presence of specific microbes.

\section{Serum concentrations of $\mathrm{HMGB1}$}

We did not find statistical significant differences between NC $(28 \pm 18 \mathrm{ng} / \mathrm{mL}$, mean and SD) vs. CRS (mean $33 \pm 15 \mathrm{ng} / \mathrm{mL}$ ) in terms of the HMGB1 levels in patients' sera.

\section{Discussion}

Emerging data indicate that chronic inflammation is associated with the CRS development and progression. A complex set of innate and adaptive immune pathways are active at the mucosal surfaces both constitutively and in response to specific antigens. Overactivity or dysregulation of mucosal immune mechanisms could lead to persistent inflammation. To the best of our knowledge, expression and function of HMGB1-RAGE in mucosal immunity of the sinonasal tract has yet not been investigated. It is known that biofilm persistence was suggested to correlate with epithelial damage, subepithelial inflammatory cell infiltration, and tumor necrosis factor alpha (TNF- $\alpha$ ) receptor expression in CRS [28]. This might result in apoptosis or hypoxia and necrotic cell death within the CRS tissue, followed by the release of HMGB1. It is likely that HMGB1 triggers paracrine activation of RAGE expressed in the CRS tissue but not (or only weakly) in normal mucosa.

Current evidences indicate that HMGB1 plays a pivotal role in the activation of innate immunity followed by increased cytokine production, development of inflammation and adaptive immune response [21-24]. Most studies of HMGB1 expression and function are focused on immune cells and suggest 

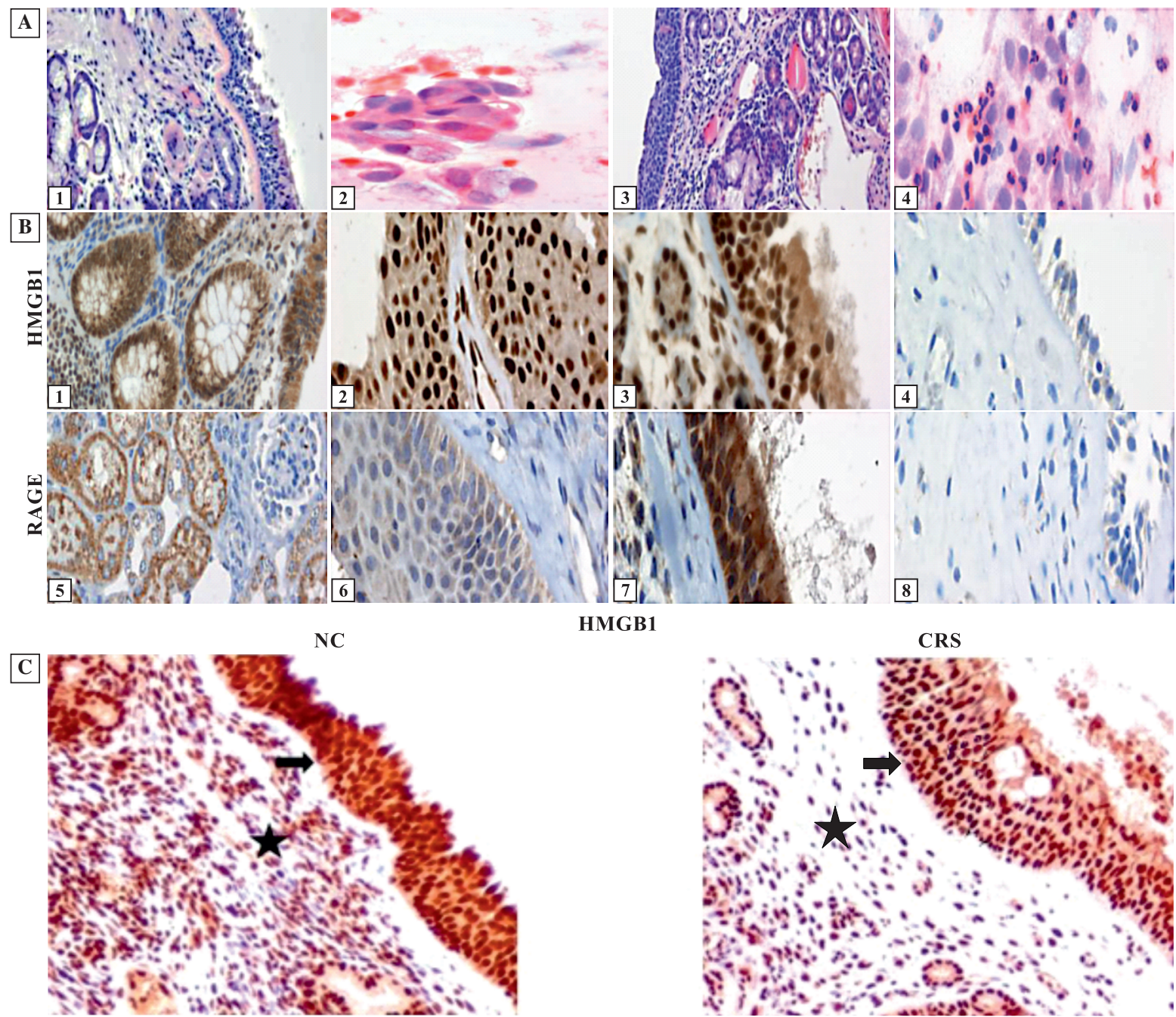

HMGB1

$\mathbf{D}$

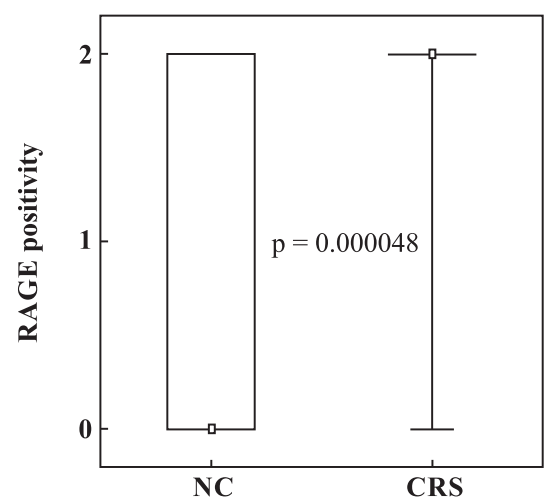

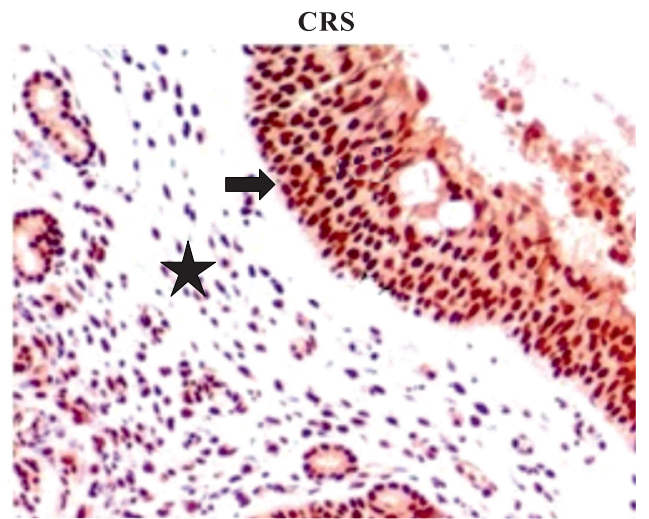

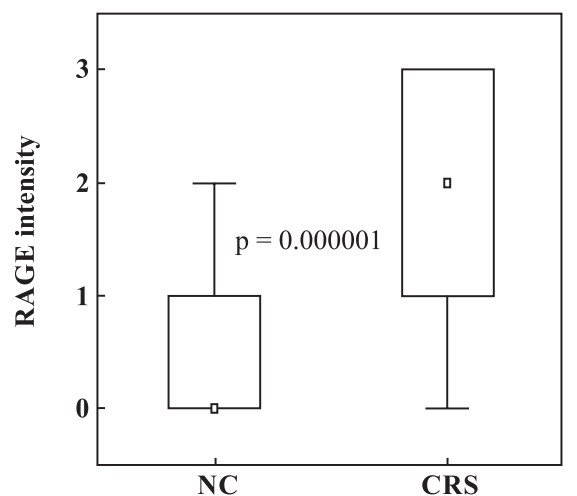

Figure 1. HMGB1 and RAGE expression in normal control (NC) and in chronic rhinosinusitis tissues (CRS). A. Hematoxylin and eosin (H \& E) staining: A1, NC mucosa; A2, NC cytology taken from under the middle turbinate; A3, CRS mucosa; A4, CRS cytology taken from under the middle turbinate; B1-4. HMGB1 expression: B1, human colon (positive control); B2, NC tissue of human nasal mucosa; B3, CRS tissue; B4, human respiratory epithelium, negative control (no primary antibody); B5-8. RAGE expression: B5, in the human kidney (positive control); B6, NC tissue; B7, CRS tissue; $\mathrm{B} 8$, isotype negative control staining in CRS; C. Intensity of HMGB1 staining in both the epithelium lining (marked with arrows) and the stroma of sinus mucosa (marked with stars). Representative pictures are shown; D. Comparison of RAGE positivity and intensity between NC and CRS patients. The sections were scored according to the \% of rhinosinusitis tissue staining (POSITIVITY) $(<25 \%=0 ; 25-75 \%=1$; and $>75 \%=2)$. The level of staining intensity was recorded as none $=0$, weak $=1$, moderate $=2$, or strong $=3$ ) (as described in Material and Methods). Representative pictures are shown. Abbreviations: NC — normal control group; CRS — patients with chronic rhinosinusitis without nasal polyps; HMGB1 - high motility group box 1; RAGE - receptor for advanced glycation end products. Magnifications: A1 and A3 $-\times 100$, all other microphotographs $-\times 400$ 

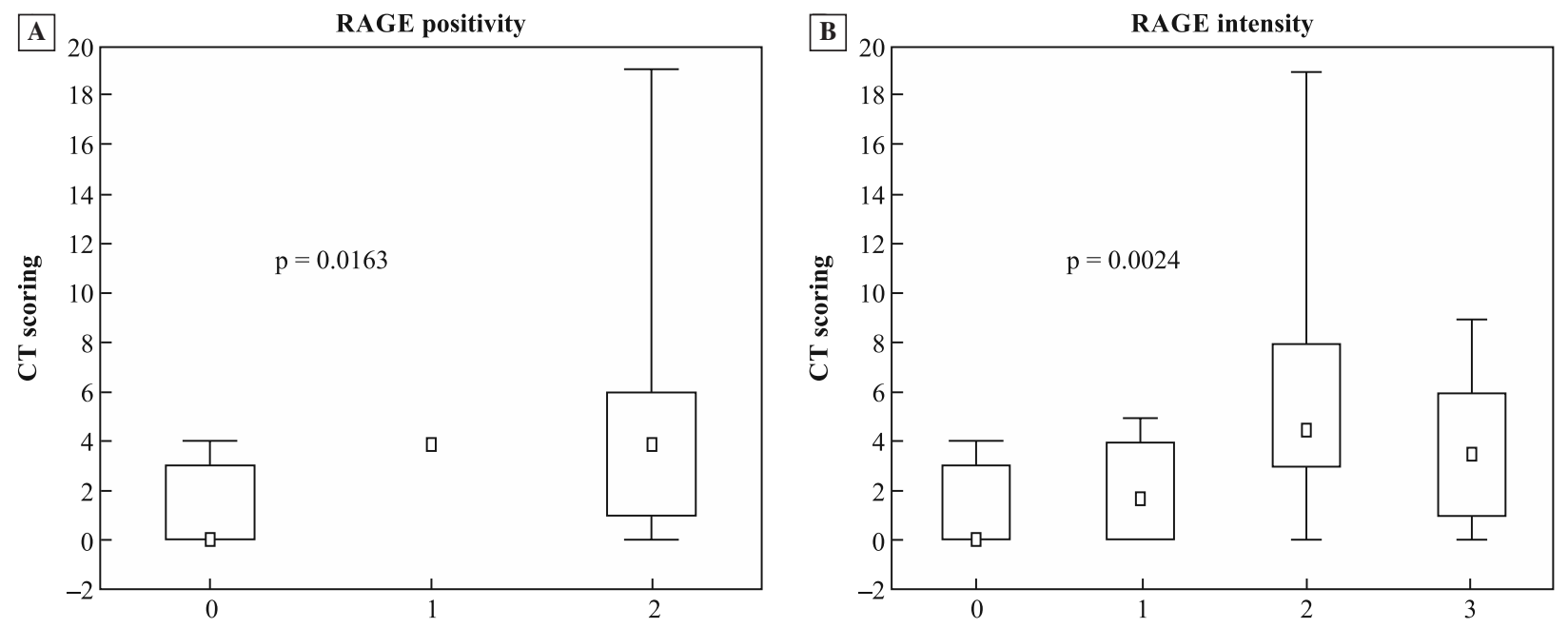

Figure 2. Association of RAGE expression with disease severity. A. RAGE positivity vs. CT scoring; B. RAGE intensity vs. CT scoring. Abbreviations as in the description of Figure 1. CT - computed tomography. Adjustments to p-values were made using the Bonferroni step-down procedure

that enhancement of HMGB1 functions promotes inflammation. We investigated expression of HMGB1 in the whole tissue including the epithelial cells and the stroma of sinonasal mucosa obtained from CRS patients and normal control subjects. Available data indicated HMGB1 expression mainly in the inflammatory infiltrates (especially eosinophilic ones), not in epithelial cells [21]. Moreover, significant differences in the HMGB1 protein level as well as the messenger RNA levels of $H M G B 1$ were found between non-eosinophilic CRSwNP and controls [29]. This observation is consistent with our findings.

Our study is interesting especially for elaborating co-expression of HMGB1 and RAGE in CRS. Consistent with the previous studies [21-24] we now confirm the expression of HMGB1 in CRS. In addition, we found that RAGE expression was significantly up-regulated in CRS compared to normal mucosal epithelium. In the microenvironment of CRS, RAGE/ /HMGB1 signaling might be, at least in part, responsible for the induction of inflammatory reactions which contribute to the disease development.

To address this hypothesis, we evaluated by immunohistochemistry the co-expression of HMGB1 and its multiligand receptor RAGE in cells of the sinonasal mucosa. Here, we report for the first time that HMGB1 and RAGE are co-expressed in CRS. Our findings are consistent with those of Ferhani et al. who found elevated RAGE level in the lung tissue of patients with chronic obstructive pulmonary disease (COPD) [30]. It was reported by Crombruggen et al. that RAGE was expressed in the human upper airways under normal physiological conditions [14]. In their
CRSsNP tissue, the tissue levels of the soluble RAGE (sRAGE) protein fraction was significantly higher compared to normal tissue, while conversely, the mRAGE (membrane-bound RAGE) levels were significantly lower compared to normal tissue. These authors hypothesized that the decrease of mRAGE protein observed in pathologic conditions was likely to be mediated via the cleavage of mRAGE by deregulated metalloproteinase activity to form sRAGE that subsequently binds cellular and extracellular matrix components [14]. Since in our study we used antibody which recognized both mRAGE and SRAGE we were unable to distinguish precisely both forms of this receptor.

Furthermore, to explore the possibility of an autocrine interaction between HMGB1 and RAGE in CRS and normal mucosa we performed immunohistochemical analysis that detected HMGB1 expression in all CRSsNP sinus mucosa tissues and normal mucosa. However, we did not find any statistical significant difference between sinus mucosa tissues and normal mucosa in terms of HMGB1 localization or staining intensity. Some authors evaluated separately the expression of HMGB1 or RAGE so they could not assess their co-expression in human upper airways tissues [22, 23]. Based on the analyses of RAGE-DNA complex structure, other authors claimed that HMGB1 is not essential for the RAGE-DNA binding event, as DNA can directly bind to RAGE in the absence of HMGB1 [31]. This observation could explain the differences between RAGE and HMGB1 expression noticed in our study.

Taken together, current literature suggests that RAGE has both pro- and anti-inflammatory prop- 

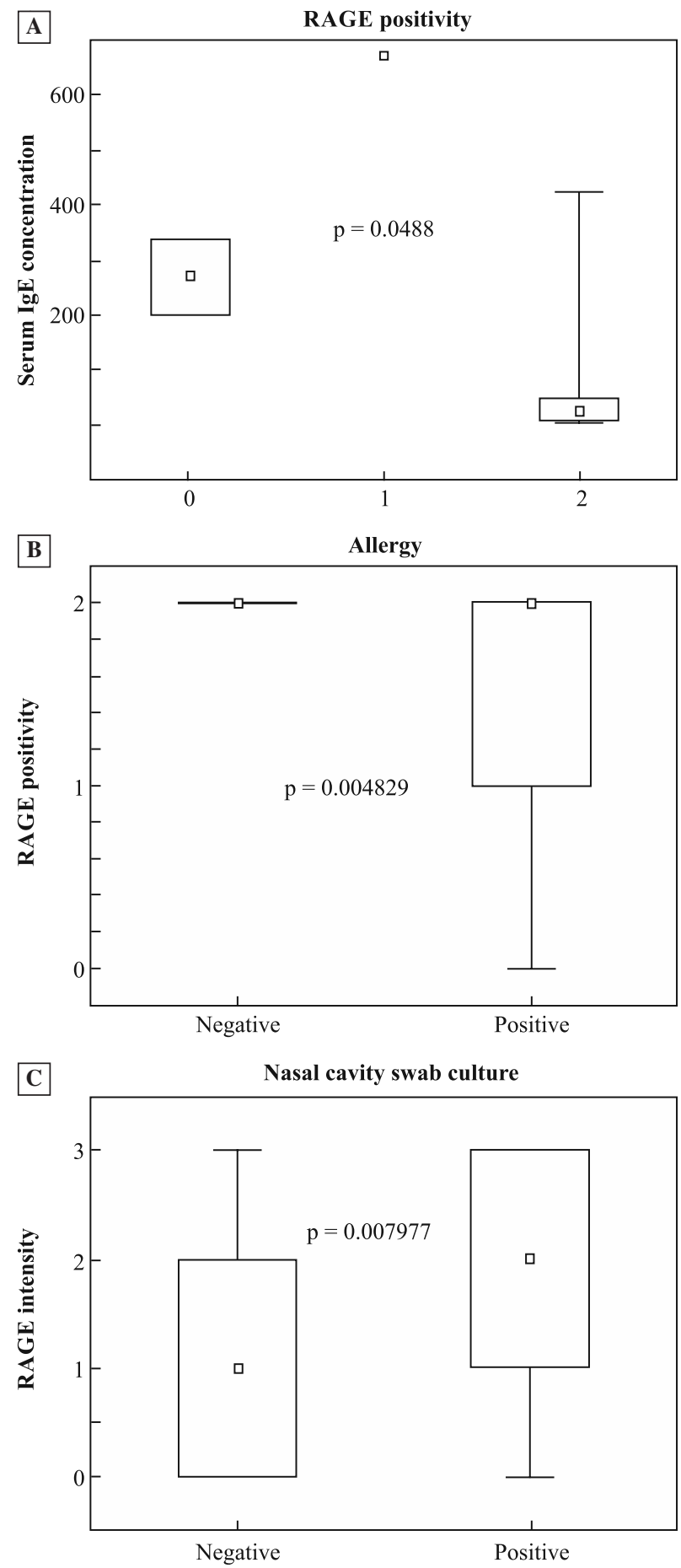

Figure 3. Comparison of negative or positive RAGE expression in CRS patients in relation to the levels of IgE, presence of allergy and microbiological content of nasal swabs. A. RAGE positivity $v s$. serum IgE concentration (IU/mL); B. RAGE positivity $v$ s. presence of allergy; C. RAGE intensity $v s$. nasal cavity swab culture

erties, but because the RAGE pathway is regulated differently in different diseases, interpretation of RAGE activation can be biased. Chavakis et al. [8] demonstrated that RAGE can directly regulate leukocyte recruitment by serving as a counter-receptor for $\beta 2$-integrin Mac-1. Further, RAGE shed and eliminated from lungs and sinonasal tissue might lose its local regulatory function that is necessary for normal immunologic homeostasis of the airways.

Since RAGE is involved in several inflammatory processes we wondered if there is any correlation between the RAGE expression and disease severity. To address this question, we analyzed the clinical relevance of RAGE and HMGB1 expression in CRS patients. We found that the level of RAGE expression correlated with disease severity. We observed significant differences in RAGE staining intensity and immunopositivity depending on CT scan results graded by the Lund-Mackay scoring system, IgE value, microbiological status, and allergy. Our data point at correlation of RAGE expression and higher CT score reflecting more extensive inflammatory changes in sinuses. Our findings prove that no correlation could be found between RAGE expression and disease severity measured by SNOT-20 questionnaire, nasal endoscopy and a number of previously performed surgeries in CRS patients. Surprisingly, cytology results, asthma or NSAIDs also did not affect RAGE expression. We also observed that a positive microbiological status of the nasal cavity correlated with higher RAGE expression which is consistent with study of Crombruggen [14], however, in our study there was no evident correlation between specific microbes and RAGE expression.

Based on our findings, we suggest that the HMGB1 and RAGE interactions might play a role in recalcitrant CRS and promote inflammatory mucosal changes. The association between RAGE expression and disease severity suggests that the HMGB1 and RAGE dysregulation may disturb the immune barrier function of the nasal mucosa. This may compromise the host defense, making the sinus mucosa more susceptible to antigenic insults, which could lead to chronic inflammation.

The potential pathogenic role of HMGB1 and RAGE in various inflammatory disorders is supported by experimental studies which demonstrate that neutralizing or binding blocking HMGB1-RAGE signaling are effective in preventing chronic inflammation [32-34]. Application of antibodies blocking HMGB1-RAGE could be also effective in CRS, enlarging the repertoire of potential therapeutic options for this disease. Treatment with selected anti-HMGB1-RAGE antibodies might attenuate persistent Th2-biased inflammation and thus immunomodulate CRS. 


\section{Acknowledgments}

We thank Dr. Miroslaw Szczepanski, Dr. Magis Mandapathil, Andrzej Kluk and Michal Piotrkowicz for technical assistance.

\section{Authors' contribution}

Conceived and designed the experiments: KD, MZ. Performed the experiments: KD, MZ. Analyzed the data: KD, MZ, MMG. Contributed reagents/materials/analysis tools: AK, MMG, MJS. Wrote the paper: $\mathrm{KD}, \mathrm{MZ}$, MMG.

\section{References}

1. Ulloa LD, Messmer D. High-mobility group box 1 (HMGB1) protein: friend and foe. Cytokine Growth Factor Rev. 2006;17:189-201. PMID: 16513409.

2. Yang H, Antoine DJ, Andersson U, Tracey KJ. The many faces of HMGB1: molecular structure-functional activity in inflammation, apoptosis, and chemotaxis. J Leukoc Biol. 2013;93:865-873. doi: 10.1189/jlb.1212662.

3. Sims GP, Rowe DC, Rietdijk ST, Herbst R, Coyle AJ. HMGB1 and RAGE in inflammation and cancer. Annu Rev Immunol. 2010;28:367-388. doi: 10.1146/annurev.immunol.021908.132603.

4. Singh VP, Bali A, Singh N, Jaggi AS. Advanced glycation end products and diabetic complications. Korean J Physiol Pharmacol. 2014;18:1-14. doi: 10.4196/kjpp.2014.18.1.1.

5. Salahuddin P, Rabbani G, Khan RH. The role of advanced glycation end products in various types of neurodegenerative disease: a therapeutic approach. Cell Mol Biol Lett. 2014; 19:407-437. doi: 10.2478/s11658-014-0205-5.

6. Park JS, Gamboni-Robertson F, He Q et al. High mobility group box 1 protein interacts with multiple Toll-like receptors. Am J Physiol Cell Physiol. 2006;290:917-924. PMID: 16267105.

7. Andersson UG, Tracey KJ. HMGB1, a pro-inflammatory cytokine of clinical interest: introduction. J Intern Med. 2004;255:318-319. PMID: 14871455.

8. Chavakis T, Bierhaus A, Al-Fakhri N et al. The pattern recognition receptor (RAGE) is a counterreceptor for leukocyte integrins: a novel pathway for inflammatory cell recruitment. J Exp Med. 2003;198:1507-1515. PMID: 14623906.

9. Bassi R, Giussani P, Anelli V et al. HMGB1 as an autocrine stimulus in human T98G glioblastoma cells: role in cell growth and migration.J Neurooncol. 2008;87:23-33. PMID: 17975708.

10. Andersson U, Erlandsson-Harris H. HMGB1 is a potent trigger of arthritis. J Intern Med. 2004;255:344-350. PMID: 14871458.

11. Mantell LL, Parrish WR, Ulloa L. HMGB-1 as a therapeutic target for infectious and inflammatory disorders. Shock. 2006;25:4-11. PMID: 16369179.

12. Andersson U, Tracey KJ. HMGB1 is a therapeutic target for sterile inflammation and infection. Annu Rev Immunol. 2011;29:139-162. doi: 10.1146/annurev-immunol-030409$-101323$.

13. Schierbeck H, Lundbäck P, Palmblad K. Monoclonal anti -HMGB1 (high mobility group box chromosomal protein 1) antibody protection in two experimental arthritis models. Mol Med. 2011;17:1039-1044. doi: 10.2119/molmed.2010.00264.

14. Van Crombruggen K, Holtappels G, De Ruyck N, Derycke L, Tomassen $\mathrm{P}$, Bachert C. RAGE processing in chronic airway conditions: involvement of Staphylococcus aureus and ECP. J Allergy Clin Immunol. 2012;129:1515-1521. doi: 10.1016/j. jaci.2012.02.021.

15. Sakurai S, Yamamoto Y, Tamei H et al. Development of an ELISA for esRAGE and its application to type 1 diabetic patients. Diabetes Res Clin Pract. 2006;73:158-165. PMID: 16488505

16. Huvenne W, van Bruaene N, Zhang N et al. Chronic rhinosinusitis with and without nasal polyps: what is the difference? Curr Allergy Asthma Rep. 2009;9:213-220. PMID: 19348721.

17. Fokkens WJ, Lund VJ, Mullol J et al. EPOS 2012: European position paper on rhinosinusitis and nasal polyps 2012. A summary for otorhinolaryngologists. Rhinology. 2012;50:1-12. doi: 10.4193/Rhino50E2.

18. Lund VJ, Kennedy DW. Staging for rhinosinusitis. Otolaryngol Head Neck Surg. 1997;117(3 Pt 2):35-40. PMID: 9334786.

19. Kenny TJ, Duncavage J, Bracikowski J, Yildirim A, Murray JJ, Tanner SB. Prospective analysis of sinus symptoms and correlation with paranasal computed tomography scan. Otolaryngol Head Neck Surg. 2001;125:40-43. PMID: 11458212.

20. Bhattacharyya T, Piccirillo J, Wippold FJ 2nd. Relationship between patient-based descriptions of sinusitis and paranasal sinus computed tomographic findings. Arch Otolaryngol Head Neck Surg. 1997;123:1189-1192. PMID: 9366698.

21. Bellussi LM, Iosif C, Sarafoleanu C et al. Are HMGB1 protein expression and secretion markers of upper airways inflammatory diseases? J Biol Regul Homeost Agents. 2013;27:791-804. PMID: 24152844.

22. Bellussi LM, Passali D, Chen L et al. LPS may enhance expression and release of HMGB1 in human nasal epithelial cells in vitro. Acta Otorhinolaryngol Ital. 2013;33:398-404. PMID: 24376296.

23. Passali D, Kern E, Lei Chen R, Bellussi L. High mobility group box 1 (HMGB 1): a new protein in the pathogenesis of ENT inflammatory and infectious diseases. Acta Otorhinolaryngol Ital. 2012;32:46-47. PMID: 22500067.

24. Hong SM, Cho JS, Um JY et al. Increased expression of high-mobility group protein $\mathrm{B} 1$ in chronic rhinosinusitis. $\mathrm{Am}$ J Rhinol Allergy. 2013;27:278-282. doi: 10.2500/ajra.2013. 27.3909.

25. Hopkins C, Browne JP, Slack R, Lund V, Brown P. The Lund-Mackay staging system for chronic rhinosinusitis: how is it used and what does it predict? Otolaryngol Head Neck Surg. 2007;137:555-561. PMID: 17903570.

26. Canakcioglu S, Tahamiler R, Saritzali G et al. Evaluation of nasal cytology in subjects with chronic rhinitis: a 7-year study. Am J Otolaryngol. 2009;30:312-317. PMID: 19720248.

27. Szczepanski MJ, Czystowska M, Szajnik M et al. Triggering of Toll-like receptor 4 expressed on human head and neck squamous cell carcinoma promotes tumor development and protects the tumor from immune attack. Cancer Res. 2009;69:3105-3113. doi: 10.1158/0008-5472.CAN-08-3838.

28. Karosi T, Csomor P, Sziklai I. Tumor necrosis factor-alpha receptor expression correlates with mucosal changes and biofilm presence in chronic rhinosinusitis with nasal polyposis. Laryngoscope. 2012;122:504-510. PMID: 22231697.

29. Chen D, Mao M, Bellussi LM, Passali D, Chen L. Increase of high mobility group box chromosomal protein 1 in eosinophilic chronic rhinosinusitis with nasal polyps. Int Forum Allergy Rhinol. 2014;4:453-462. PMID: 24504744.

30. Ferhani N, Letuve S, Kozhich A et al. Expression of high -mobility group box 1 and of receptor for advanced glycation end products in chronic obstructive pulmonary disease. Am J Respir Crit Care Med. 2010;181:917-927. PMID: 20133931. 
31. Sirois CM, Jin T, Miller AL et al. RAGE is a nucleic acid receptor that promotes inflammatory responses to DNA. J Exp Med. 2013;210:2447-2463. doi: 10.1084/jem.20120 201.

32. Ostberg T, Kawane K, Nagata S et al. Protective targeting of high mobility group box chromosomal protein 1 in a spontaneous arthritis model. Arthritis Rheum. 2010;62:2963-2972. doi: 10.1002/art.27590.
33. Pisetsky DS, Erlandsson-Harris H, Andersson U. High-mobility group box protein 1 (HMGB1): an alarm in mediating the pathogenesis of rheumatic disease. Arthritis Res Ther. 2008;10:209. doi: 10.1186/ar2440.

34. Lutterloh EC, Opal SM, Pittman DD et al. Inhibition of the RAGE products increases survival in experimental models of severe sepsis and systemic infection. Crit Care. 2007;11:122. PMID: 18042296.

Submitted: 19 July, 2014 Accepted after reviews: 12 March, 2015 Available as AoP: 13 March, 2015 